\title{
Hadron masses from dynamical, non-perturbatively $O(a)$ improved Wilson fermions
}

\author{
H. Stüben ${ }^{\mathrm{a}}, Q C D S F$ and $U K Q C D$ Collaborations \\ ${ }^{a}$ Konrad-Zuse-Zentrum für Informationstechnik Berlin, 14195 Berlin, Germany
}

We present results on light hadron masses from simulations of full QCD and report on experiences in running such simulations on a Hitachi SR8000-F1 supercomputer.

\section{INTRODUCTION}

With the installation of a 112 node Hitachi SR8000-F1 at the Leibniz-Rechenzentrum in Munich the QCDSF Collaboration has started simulating QCD with two degenerate flavors of fermions. In a cooperative program with the UKQCD Collaboration (see talk by Irving [1]) we share configurations giving both collaborations the possibility to do measurements at a larger set of bare parameters.

This contribution has two parts. In the first part we report on performance of QCD calculations on the Hitachi SR8000-F1. In the second part we present results on the light hadron mass spectrum for dynamical, non-perturbatively $O(a)$ improved Wilson fermions. We focus on comparison with our quenched results [2, 3].

\section{QCD ON THE HITACHI SR8000-F1}

\subsection{Hardware overview}

The SR8000 in Munich is a "cluster" of 112 symmetric multiprocessor nodes. Each node has 8 CPUs and (at least) 8 GByte of shared memory. Each CPU has a a 128 kByte 4 -way associative cache, 160 registers and has a pseudovectorization facility. The peak performance of a $\mathrm{CPU}$ is $1.5 \mathrm{Gflop} / \mathrm{s}$ giving a peak performance of $1344 \mathrm{Gflop} / \mathrm{s}$ for the whole machine.

\subsection{Pseudo-vectorization}

It order to get good performance it is essential to employ pseudo-vectorization. At the hardware level pseudo-vectorization is implemented by efficient pre-fetch and pre-load mechanisms. At the programming level the compiler tries to vectorize most inner loops. These loops are vectorized under the usual conditions, i.e., if there are no data access conflicts, no function/subroutine calls and no if-blocks in the loop body.

On the SR8000 one can basically use the same code as on a standard RISC processor and more important one can have the same data layout as for a RISC CPU. For example our data layout is (in Fortran 90 notation)

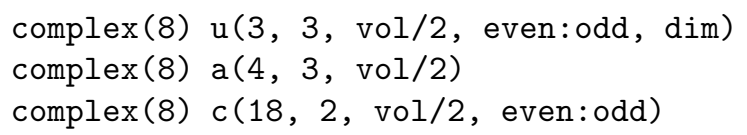

for the gauge, pseudo-fermion and clover field. In contrast to a real vector computer the vector index does not have to be shifted to the first place.

\subsection{Single CPU performance}

To fix the notation let $M$ be the fermion matrix, $D$ the Wilson hopping term and $A$ the clover term:

$$
\begin{aligned}
& M=A-\kappa D \\
& A=1-\kappa c_{\mathrm{Sw}} \sigma_{\mu \nu} \mathcal{F}_{\mu \nu}
\end{aligned}
$$

Performance figures for the multiplication of $D$, $A, A^{-1}$ and $M$ with a vector are given in Table 1. These figures were obtained with Fortran 90 code using 64 bit arithmetic on an $8^{4}$ lattice. The code performs well in comparison with RISC processors where one typically sustains $10-20 \%$ of the peak performance.

\subsection{Parallelization}

Our code is parallelized with MPI. In addition we have experimented with a hybrid program- 

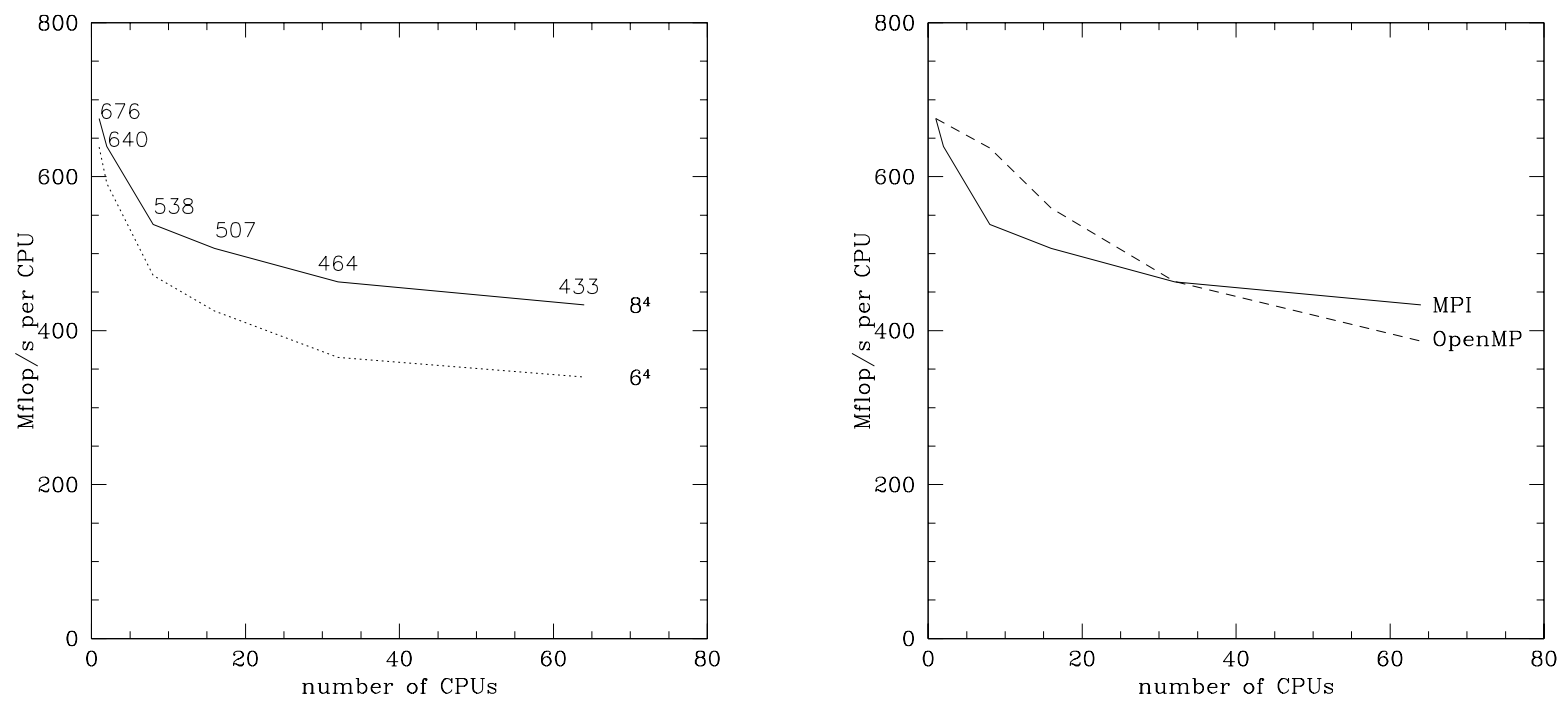

Figure 1. Communication loss for constant lattice volume per CPU as indicated. Further explanations are given in the text.

Table 1

Performance of the multiplication with a vector on a single CPU.

\begin{tabular}{crr}
\hline$D$ & $640 \mathrm{Mflop} / \mathrm{s}$ & $(43 \%$ of peak $)$ \\
$A$ & $1160 \mathrm{Mflop} / \mathrm{s}$ & $(77 \%$ of peak $)$ \\
$A^{-1}$ & $630 \mathrm{Mflop} / \mathrm{s}$ & $(42 \%$ of peak $)$ \\
$M$ & $676 \mathrm{Mflop} / \mathrm{s}$ & $(45 \%$ of peak $)$ \\
\hline
\end{tabular}

ming model using OpenMP for distributing the work over the CPUs of a single node and MPI for the communication between nodes. Figure 1 shows the communication loss for the fermion matrix multiplication.

The solid lines (being identical in both plots) show performance measurements for a lattice volume of $8^{4}$ per CPU for the pure MPI code. In the left plot these are compared with measurements on volumes of $6^{4}$ per CPU. In the right plot comparison is done with the hybrid programming model again on a CPU local volume of $8^{4}$.

As can be seen from Figure 1 the communication loss is quite substantial. The observations on the pure MPI code can be understood qualitatively in the following way. Using more and more CPUs the communication pattern becomes more and more complex. Within one node when using 2,4 or 8 CPUs there are 2,4 or 6 boundaries which have to be communicated and the surface/volume ratio is $2 / 8,4 / 8$ or $6 / 8$ respectively.

Between nodes communication is along 1,2 or 3 dimensions (in our program the lattice can be decomposed maximally in three dimensions) when using 2,4 , or 8 (and more) nodes leading again to more and more complicated communication patterns.

On a single node parallelization with OpenMP is more efficient than with MPI. This is to be expected because there is no communication needed in that case. But surprisingly this gain does not persist when switching on inter node communication. In both cases the same amount of data has to be transferred between the nodes. But in the pure MPI version more calls are needed to copy data between nodes and in addition data has to be copied within the nodes. Both should lead to larger communication overhead.

According to the hardware performance monitor we are running at about $400-450 \mathrm{Mflop} / \mathrm{s}$ per $\mathrm{CPU}$ in our production runs. This is in accordance with the $433 \mathrm{Mflop} / \mathrm{s}$ given in Figure 1. 


\section{LIGHT HADRON MASSES}

\subsection{Simulation details}

We investigate full QCD with the standard Wilson action for the plaquette and the fermions plus the $O(a)$ improvement term. For the improvement coefficient $c_{\mathrm{sw}}(\beta)$ we use the nonperturbative values determined by the ALPHA collaboration 4 .

Simulation parameters and statistics are listed in Table 2. In these simulations the $m_{\mathrm{PS}} / m_{\mathrm{V}}$ ratio lies between 0.60 and 0.83 . The Sommer scale [5] $r_{0} / a$ varies between 4.7 and 5.5 [1].

Table 2

\begin{tabular}{crrr}
\hline$\beta$ & $\kappa_{\text {sea }}$ & $V$ & $N_{\text {traj }}$ \\
\hline \multicolumn{4}{c}{ UKQCD } \\
\hline 5.20 & 0.1350 & $16^{3} \times 32$ & 6000 \\
& 0.1355 & $16^{3} \times 32$ & 8000 \\
5.26 & 0.1345 & $16^{3} \times 32$ & 4000 \\
5.29 & 0.1340 & $16^{3} \times 32$ & 4000 \\
\hline \multicolumn{4}{c}{ QCDSF } \\
\hline 5.29 & 0.1350 & $16^{3} \times 32$ & 2270 \\
& 0.1355 & $24^{3} \times 48$ & 605 \\
\hline
\end{tabular}

Figure 2 shows the integrated autocorrelation time of the plaquette $\tau_{\text {int }, P}(t)=\frac{1}{2}+\sum_{t^{\prime}=1}^{t} \rho_{P}\left(t^{\prime}\right)$ ( $\rho_{P}$ is the normalized autocorrelation function) for the QCDSF run on the $16^{3} \times 32$ lattice. From the plot we read off that in this case $\tau_{\text {int }, P} \lesssim 10$.

\subsection{Results}

Figure 3 shows an APE plot. For clarity error bars for the quenched results $(0.1-3.5 \%)$ are not shown. One sees that for currently reachable sea quark masses unquenching has, if any, only small effects on the light hadron spectrum and that with dynamical fermions it is a long way to $m_{\mathrm{PS}} / m_{\mathrm{V}}$ ratios as small as in the quenched case.

In the following we compare our dynamical results with chiral extrapolations done in the quenched case at $\beta=6.0$ where $r_{0} / a=5.36$. This is within the range of $r_{0} / a$ of the dynamical runs studied. Let us first recall the chiral extrapolation of $m_{\mathrm{V}}$. Our quenched results for $m_{\mathrm{V}}$ are very well described by a phenomenological fit

$$
\left(a m_{\mathrm{V}}\right)^{2}=\left(a M_{\mathrm{V}}\right)^{2}+b_{2}\left(a m_{\mathrm{PS}}\right)^{2}+b_{3}\left(a m_{\mathrm{PS}}\right)^{3}
$$

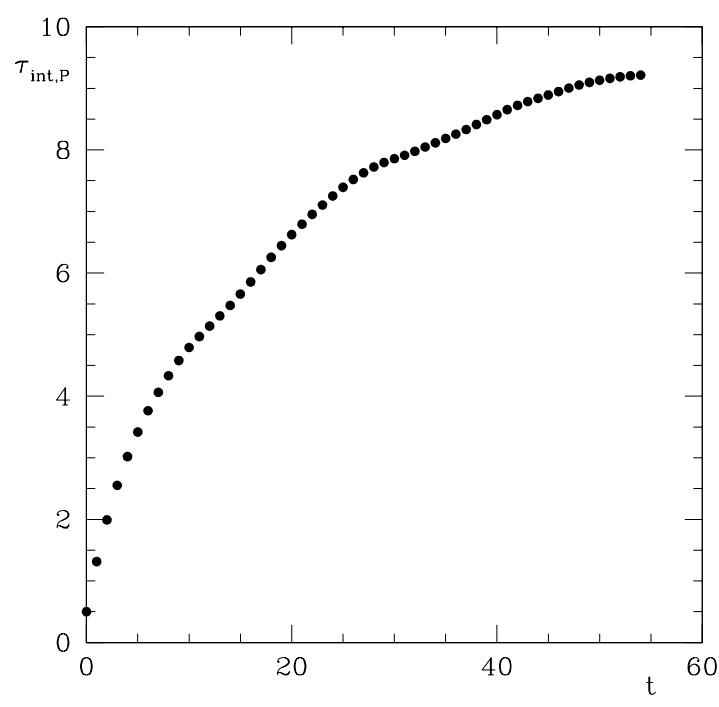

Figure 2. Integrated autocorrelation time of the plaquette for the run at $\beta=5.29$ and $\kappa=0.1350$.

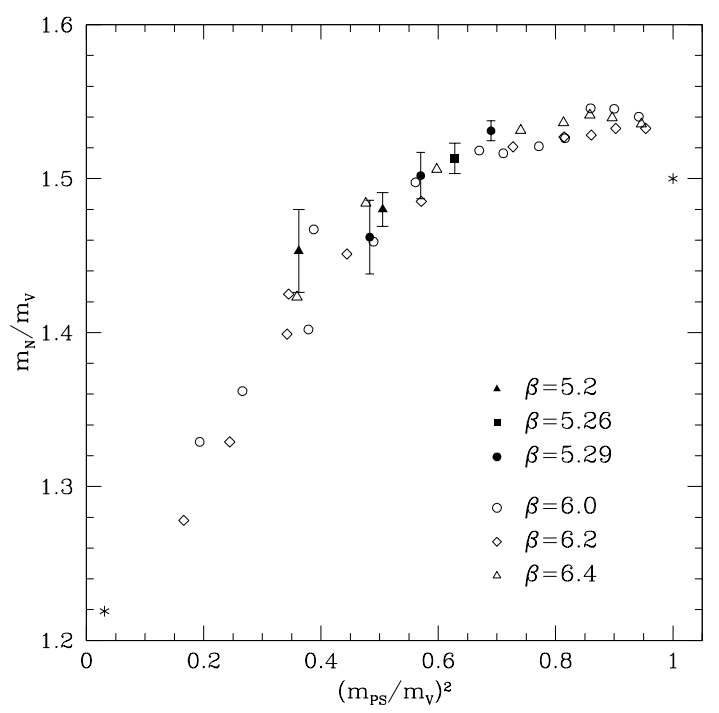

Figure 3. APE plot. Open symbols represent our quenched results [2,3] without error bars. Filled symbols are our dynamical results.

where $b_{2}=0.910(20)$ and $b_{3}=0.049(15)$ [3]. The lightest three points were excluded from the fit. The deviation of these points is believed to be a quenching artifact [2,3]. A quenching artifact appearing in quenched chiral perturbation theory for the vector meson mass is a term $\propto a m_{\mathrm{PS}}[6]$. 


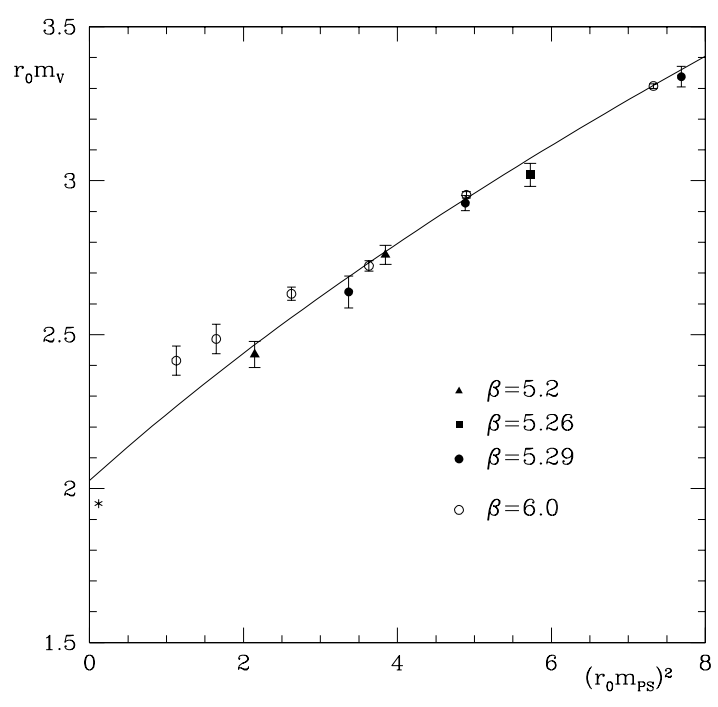

Figure 4. Comparison of quenched data and fit (3) with the dynamical results. The star indicates the experimental value.

In Figure 4 we plotted our quenched results together with the dynamical ones. The line represents the fit (3). The fit range is much larger than the range of this plot which only includes the six smallest masses (open symbols). Towards the chiral limit we see that the dynamical data (filled symbols) lie systematically below the quenched data. The dynamical results are consistent with the quenched fit.

The corresponding picture for $m_{\mathrm{N}}$ is shown in Figure 5. The open symbols represent quenched results and the line is a phenomenological fit like (3) where the curvature $b_{3}$ is again small. The filled symbols are dynamical results. The agreement between quenched and dynamical results is more pronounced than for $m_{\mathrm{V}}$.

\subsection{Conclusions}

We observe that the results with nonperturbatively $O(a)$ improved dynamical fermions scale well. Although we are not able to do a continuum extrapolation yet, we see no large discretization errors. In the considered range of sea quark masses we found no clear evidence for unquenching effects in comparison with previous quenched results.

However, our results seem to indicate that the

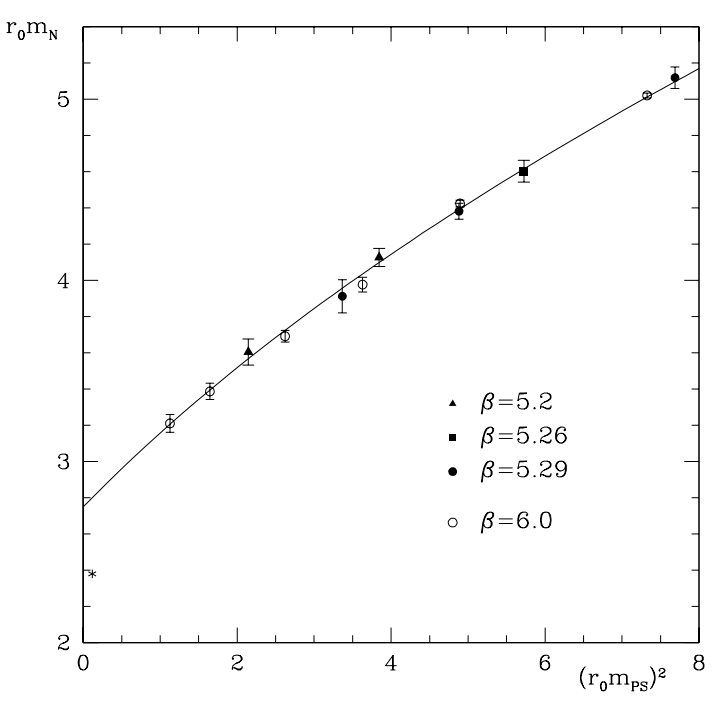

Figure 5. Comparison of $m_{\mathrm{N}}$ quenched data and fit like in Figure 4 with the dynamical results. The star indicates the experimental value.

vector meson mass $m_{\mathrm{V}}$ approaches the chiral limit in a different way. This may be another hint, that quenched artifacts are visible in the quenched data.

\section{ACKNOWLEDGEMENTS}

The computations were done on the Hitachi SR8000-F1 at LRZ (Munich), on the Cray T3Es at EPCC (Edinburgh), NIC (Jülich), (ZIB) Berlin and on the APE/Quadrics at DESY Zeuthen. We thank these institutions for support. UKQCD acknowledges PPARC grants GR/L22744 and PPA/G/S/1998/000777.

\section{REFERENCES}

1. A.C. Irving, these proceedings.

2. QCDSF Collaboration, Nucl. Phys. B (Proc. Suppl.) 83-84 (2000) 173.

3. D. Pleiter, Ph.D. thesis, Freie Universität Berlin, 1999.

4. K. Jansen and R. Sommer, Nucl. Phys. B530 (1998) 185.

5. R. Sommer, Nucl. Phys. B411 (1994) 839.

6. M. Booth, G. Chiladze and A.F. Falk, Phys. Rev. D55 (1997) 3092. 